\title{
Modeling of Ground-Penetrating-Radar Antennas With Shields and Simulated Absorbers
}

\author{
Uğur Oğuz and Levent Gürel, Senior Member, IEEE
}

\begin{abstract}
A three-dimensional (3-D) finite-difference time-domain (FDTD) scheme is employed to simulate ground-penetrating radars. Conducting shield walls and absorbers are used to reduce the direct coupling to the receiver. Perfectly matched layer (PML) absorbing boundary conditions are used for matching the multilayered media and simulating physical absorbers inside the FDTD computational domain. Targets are modeled by rectangular prisms of arbitrary permittivity and conductivity. The ground is modeled by homogeneous and lossless dielectric media.
\end{abstract}

Index Terms-Finite-difference time-domain (FDTD), groundpenetrating radar, perfectly matched layers (PMLs).

\section{INTRODUCTION}

$\mathbf{T}$ HE finite-difference time-domain (FDTD) [1] method has been one of the most popular methods for the simulation of ground-penetrating-radar (GPR) systems [2]-[4] in recent years. In contrast to integral-equation-based techniques, the FDTD method is a powerful tool to solve problems involving arbitrary inhomogeneities [5]-[11] and nonuniformly (nonplanarly) layered media. The number of unknowns required by the FDTD method does not necessarily increase as the configuration of the inhomogeneous ground model changes, as long as the resolution of the discretized problem space (i.e., the FDTD computational domain) is not changed. In this paper, three-dimensional (3-D) FDTD simulation results of GPR systems are presented for design, analysis, and evaluation purposes. A perfectly matched layer (PML) [12]-[17] absorbing boundary condition (ABC) is employed to terminate the FDTD computational domain. In addition to this usual function of the PML at the borders, in this paper, we report a novel use of the PML ABC inside the FDTD computational domain to simulate physical absorbers.

The computational model of a typical GPR problem is illustrated in Fig. 1. This model includes the following elements.

1) The ground is modeled as a lossless homogeneous dielectric medium with arbitrary permittivity. Lossy and inhomogeneous ground models are reported elsewhere [18].

2) The air is modeled as free space (vacuum).

3) The ground-air interface is planar and lies on a constant- $z$ plane. Surface roughness of the ground is considered in another study [18].

4) The targets can be arbitrary in quantity, shape, location, and material properties. In this work, targets are modeled

Manuscript received June 25, 2000; revised December 18, 2000.

The authors are with the Department of Electrical and Electronics Engineering, Bilkent University, Bilkent, Ankara, Turkey (e-mail: uoguz@cem.bilkent.edu.tr; lgurel@ee.bilkent.edu.tr).

Publisher Item Identifier S 0018-926X(01)09193-1.

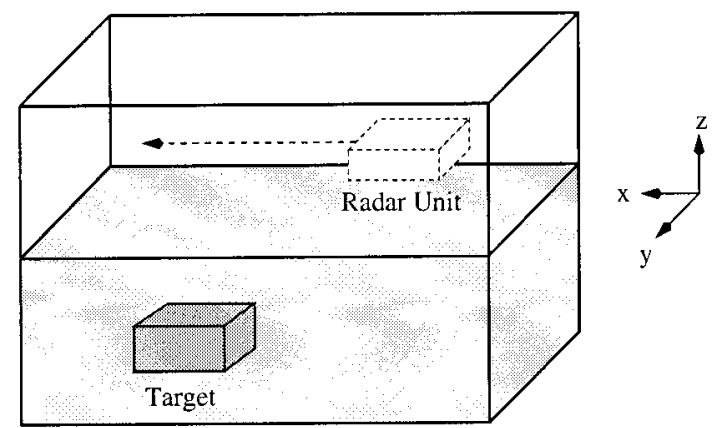

Fig. 1. Geometry of a typical GPR problem with a buried scatterer. A radar unit travels over the ground-air interface at a fixed elevation.

as dielectric and conducting rectangular prisms. Modeling results of multiple dielectric and conducting targets of prism and disk shapes are reported in other papers [18]-[20].

5) The radar unit consists of transmitting and receiving antennas and, optionally, shields and absorbers. Details of various radar-unit models will be reported in the following sections together with their respective advantages and disadvantages.

6) The computational domain is terminated with an implementation of the PML ABC that is suitable for layered media [19, Appendix].

\section{THE RADAR UNIT}

A majority of the simulated GPR models found in the literature contain a transmitting and a receiving antenna, located at the same elevation above the ground-air interface [6]-[8]. The transmitter $(\mathrm{T})$ generates the fields penetrating the ground with a particular polarization and the receiver $(\mathrm{R})$ collects and samples the fields with the same polarization. Fig. 2 depicts such a transmitter-receiver (TR) configuration.

In this work, the transmitting antenna is selected as a small $x$-polarized dipole, modeled by a single Yee cube of constant current density. The time variation of this current source is given by

$$
J_{x}(t)=\frac{1}{\Delta^{3}}\left[4\left(\frac{t}{\tau}\right)^{3}-\left(\frac{t}{\tau}\right)^{4}\right] e^{-\frac{t}{\tau}}
$$

where $\tau=1 /\left(4 \pi f_{0}\right), f_{0}$ is the center frequency of the pulse, and $\Delta=\Delta x=\Delta y=\Delta z$ is the sampling interval in space. For a center frequency of $f_{0}=500 \mathrm{MHz}$, plots of (1) in the time and frequency domains are shown in Fig. 3(a) and (b), respectively. 


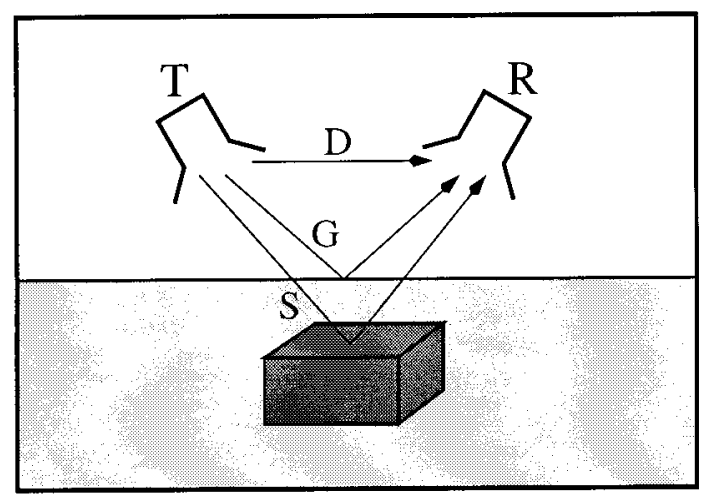

Fig. 2. The transmitter-receiver configuration of the radar unit. The total received signal is an aggregate of three signals: the direct signal (D) coupled from the transmitter to the receiver, the signal reflected from the ground $(\mathrm{G})$, and the signal scattered by the buried target (S).
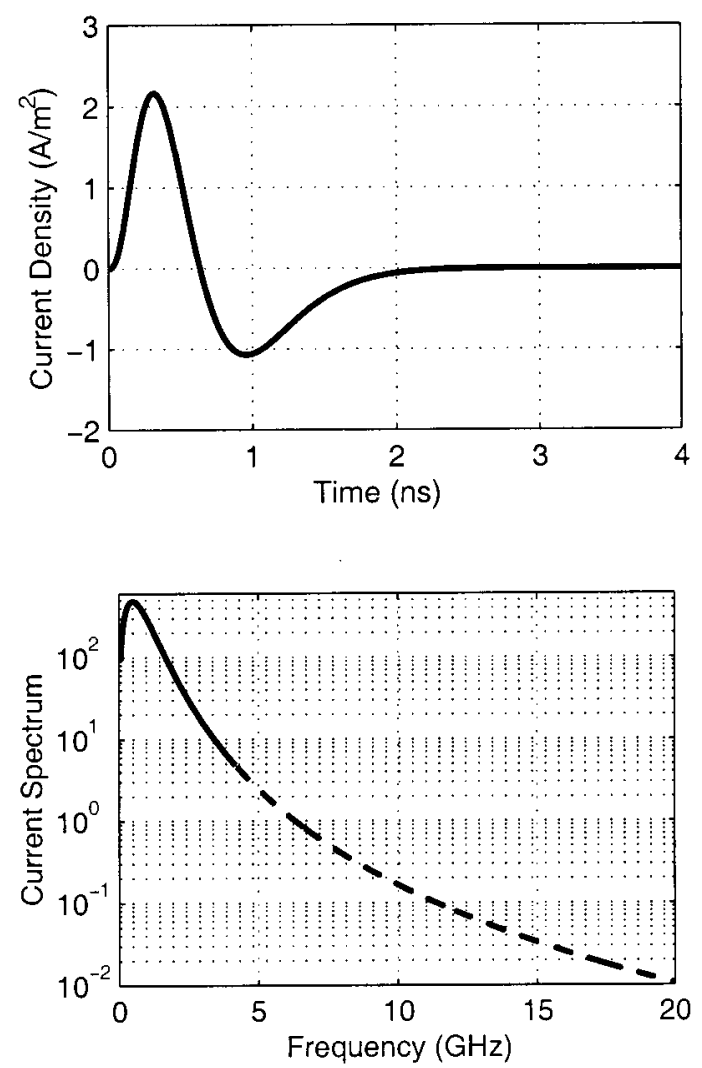

Fig. 3. (a) Time and (b) frequency variations of the current source with 500 $\mathrm{MHz}$ center frequency.

This pulse function is both time-limited and band-limited and is preferred for its small dc content and smooth character in time. Fig. 3(a) demonstrates that the source function with $500 \mathrm{MHz}$ center frequency dies out after 2 ns. In Fig. 3(b), frequency components with more than $40 \mathrm{~dB}$ (two orders of magnitude) below the largest component are plotted in dashes. It is seen that a substantial amount of energy of the source signal predominantly lies between $0-4 \mathrm{GHz}$.
This current source is coupled to the FDTD computational domain using the following one of the six Maxwell's equations:

$$
\frac{\partial E_{x}}{\partial t}=\frac{1}{\epsilon}\left(\frac{\partial H_{z}}{\partial y}-\frac{\partial H_{y}}{\partial z}-\sigma E_{x}-J_{x}\right) .
$$

The discretization of (2) yields

$$
\begin{aligned}
E_{x}^{i+\frac{1}{2}, j, k, n}= & \frac{2 \epsilon}{2 \epsilon}+\sigma \Delta t \\
& -\frac{2 \Delta t}{\Delta(2 \epsilon+\sigma \Delta t)} E_{x}^{i+\frac{1}{2}, j, k, n-1} \\
& \cdot\left(H_{y}^{i+\frac{1}{2}, j, k+\frac{1}{2}, n-\frac{1}{2}}\right. \\
& -H_{y}^{i+\frac{1}{2}, j, k-\frac{1}{2}, n-\frac{1}{2}}-H_{z}^{i+\frac{1}{2}, j+\frac{1}{2}, k, n-\frac{1}{2}} \\
& \left.+H_{z}^{i+\frac{1}{2}, j-\frac{1}{2}, k, n-\frac{1}{2}}\right) \\
+ & \frac{2 \Delta t}{(2 \epsilon+\sigma \Delta t)} J_{x}^{i+\frac{1}{2}, j, k, n-\frac{1}{2}}
\end{aligned}
$$

where $\Delta t$ is the sampling interval in time (time step). The other five equations are not modified and therefore are not repeated here for brevity. The full set of discretized Maxwell's equations can be found in many FDTD references, e.g., [21] and [22]. If the location of the transmitter is

$$
\left(x_{t}, y_{t}, z_{t}\right)=\left(i_{t} \Delta x, j_{t} \Delta y, k_{t} \Delta z\right)
$$

then $J_{x}$ in (3) is nonzero only when $(i, j, k)=\left(i_{t}, j_{t}, k_{t}\right)$.

The receiver is implemented by sampling and storing the values of the $x, y$, or $z$ component of the electric field, depending on the choice of polarization. Thus, discrete values

$$
E^{i, j, k, n}=E(i \Delta x, j \Delta y, k \Delta z, n \Delta t)
$$

of the electric-field function $E(x, y, z, t)$ are obtained at the receiver. When the radar unit is stationary and the receiver collects data at a point $\left(x_{0}, y_{0}, z_{0}\right)$ in space for successive instants of time, this process is called an A-scan and the resulting one-dimensional array of data is denoted as

$$
E^{n}=E\left(i \Delta x=x_{0}, j \Delta y=y_{0}, k \Delta z=z_{0}, n \Delta t\right) .
$$

A B-scan is obtained by performing repeated A-scan measurements at discrete points on a linear path. For example, the twodimensional (2-D) array of electric-field values

$$
E^{i, n}=E\left(i \Delta x, j \Delta y=y_{0}, k \Delta z=z_{0}, n \Delta t\right)
$$

can be considered as a set of B-scan data when the radar unit moves in the $x$ direction. Similarly

$$
E^{i, j, n}=E\left(i \Delta x, j \Delta y, k \Delta z=z_{0}, n \Delta t\right) .
$$

denotes the 3-D data collected on a 2-D rectangular grid of discrete points on a constant $z$-plane. This measurement is called a C-scan and can be considered as combining several B-scans. 


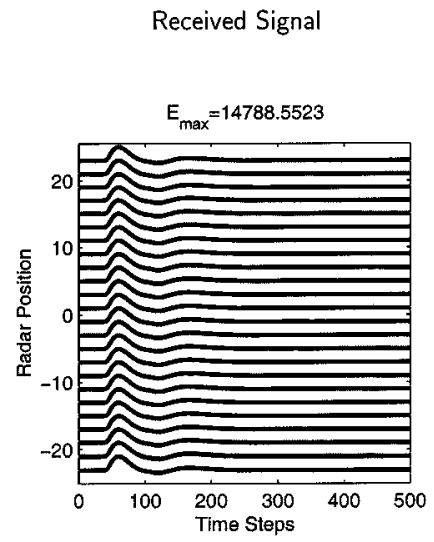

(a)

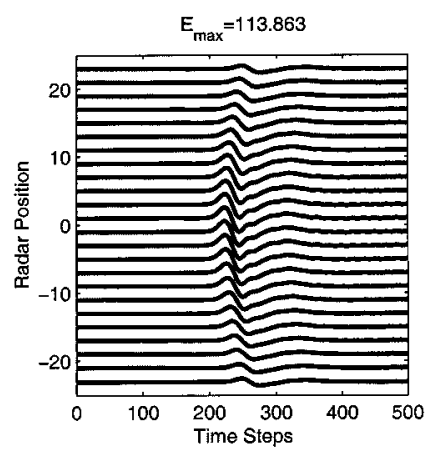

(b)
Fig. 4. Simulation results of a dielectric $\left(\epsilon_{r}=3\right)$ rectangular prism $\left(5 \times 5 \times 4 \mathrm{~cm}^{3}\right)$ buried $2.5 \mathrm{~cm}$ under the ground $\left(\epsilon_{r}=8\right)$. (a) The received and (b) the scattered signals recorded by the TR pair. The center frequency of the current signal on the transmitting dipole is $500 \mathrm{MHz}$. The unit of radar position is $\Delta$, where $\Delta=5 \mathrm{~mm}$, and the unit of time steps is $\Delta t$, where $\Delta t=9 \mathrm{ps}$. The $\mathrm{T}$ and $\mathrm{R}$ are separated by $10.5 \mathrm{~cm}$, and both are $13 \mathrm{~cm}$ above the ground.

\section{ISSUES ON GPR DESIGN AND DETECTION}

In the typical TR configuration of Fig. 2, the total received signal is the sum of three individual signals: the direct signal (D) coupled from the transmitter to the receiver, the signal reflected from the ground $(\mathrm{G})$, and the signal scattered by the buried target (S). The desired signal is the S signal, which contains information about the position and the characteristics of the buried target. However, the D signal is usually much larger than the $\mathrm{S}$ signal, rendering the detection of the $\mathrm{S}$ signal (and thus the buried object) difficult or impossible in the total received signal $(\mathrm{D}+\mathrm{G}+\mathrm{S})$. As an example, Fig. 4 displays the B-scan plots of the large $\mathrm{D}+\mathrm{G}+\mathrm{S}$ signals and the small $\mathrm{S}$ signals recorded by a radar unit moving above a dielectric rectangular prism buried under the ground-air interface. The S signals displayed in Fig. 4(b) are obtained with the subtraction of the results of an extra simulation involving a homogeneous ground in the absence of the target. The result of this extra simulation provides the sum of D and $\mathrm{G}$ signals, which are subtracted from the $\mathrm{D}+\mathrm{G}+\mathrm{S}$ signals to extract the $\mathrm{S}$ signals.

For each of the plots in Fig. 4(a) and (b), the maximum value of the electric field obtained in each B-scan measurement, i.e.,

$$
E_{\max }=\max _{i} \max _{n} E_{x}^{i, n}
$$

is displayed in the title of the corresponding plot and is used to normalize the amplitudes of the A-scan signals in that plot. Comparison of these two values and the examination of the B-scan plots in Fig. 4(a) reveals that a very large D signal renders the detection of buried targets very difficult. However, there are some special techniques that can be employed to ease the detection of the S signal. Some of these techniques are as follows.

1) The $D+G$ signal can be computed or measured exactly, or in most cases approximately, in the absence of the buried object(s), and subtracted from the total received signal to obtain the $\mathrm{S}$ signal. Obtaining the $\mathrm{D}+\mathrm{G}$ signal ex- actly is possible only when a simulation with a homogeneous ground model is performed [6],[10], as mentioned above. In actual measurements, the ground is not homogeneous, and therefore, the $\mathrm{D}+\mathrm{G}$ signal can be approximately obtained by averaging the received signals over a region, which is believed to reflect the typical environment characteristics, but with no buried target(s). Even an approximate determination of the $\mathrm{D}+\mathrm{G}$ signal facilitates the detection of the S signal remarkably, as demonstrated in [18].

2) Using short pulses with high-frequency contents, the $D$, $\mathrm{G}$, and S signals can be separated in time in the total received signal. Then, the $\mathrm{D}$ or $\mathrm{D}+\mathrm{G}$ signals can be eliminated by windowing them out in time. Fig. 5 displays the simulation results of a GPR unit operating at a center frequency of $2000 \mathrm{MHz}$. Although the D signals shown in Fig. 5(a) are much larger than the $\mathrm{S}$ signals in Fig. 5(b), they are much narrower in time and die out after 300 time steps. Therefore, by removing the first 300 time steps out of the picture, it is possible to detect the $\mathrm{S}$ signals in the magnified $\mathrm{D}+\mathrm{G}+\mathrm{S}$ signals visually, as shown in Fig. 5. It would not be useful to apply this technique on the results of Fig. 4 , where $f_{0}=500 \mathrm{MHz}$ and thus the pulse width is four times larger.

3) If the $\mathrm{D}$ and $\mathrm{S}$ signals can be separated in time using short pulses, then, as an alternative method to time windowing, the total received signal can be multiplied by a scaling function that grows exponentially in time. This way, the $\mathrm{S}$ signal can be magnified to a level that allows comfortable detection even in the presence of the D signal.

4) A transmitter-receiver-transmitter (TRT) type of GPR configuration can be used [9],[19]. If the two transmitters are fed with a phase difference of $180^{\circ}$ between them, then the D signals and most of the $G$ signals cancel out at the receiver location, due to the symmetry in the problem.

5) To substantially weaken the $D$ signal, the transmitter and the receiver can be isolated using conductive and/or absorbing shields [8],[23]. This technique is further investigated in this paper, and simulation results of such GPR designs are presented in Section IV.

The two examples depicted by Figs. 4 and 5 emphasize the visual detection of the $\mathrm{S}$ signal in the total received signal. In practice, sophisticated detection algorithms are employed instead of visual detection. For example, one of the simpler detection algorithms is used in [18]. Since algorithmic detection can be achieved even when visual detection is not possible, visual detection is not an absolute necessity. Nevertheless, the effects of weakening the D signal on the visual detection is studied in this paper since similar improvements will be observed in algorithmic detection, too. That is, a large D signal degrades the performances of detection algorithms, too, and needs to be eliminated as much as possible. Various GPR designs are presented in Section IV for this purpose.

\section{GPR MODELING With SHIELDS AND ABSORBERS}

In the previous section, isolation of the transmitting and the receiving antennas was suggested to weaken the D signal and 


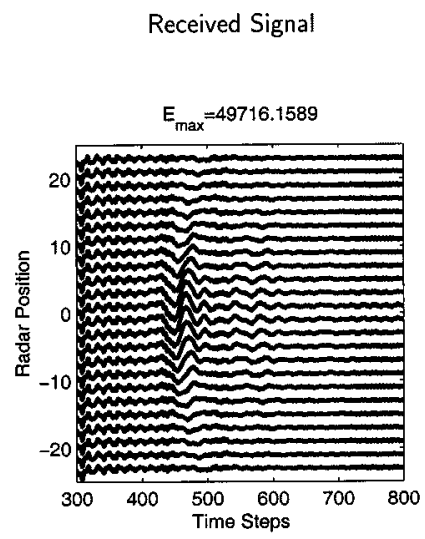

(a)

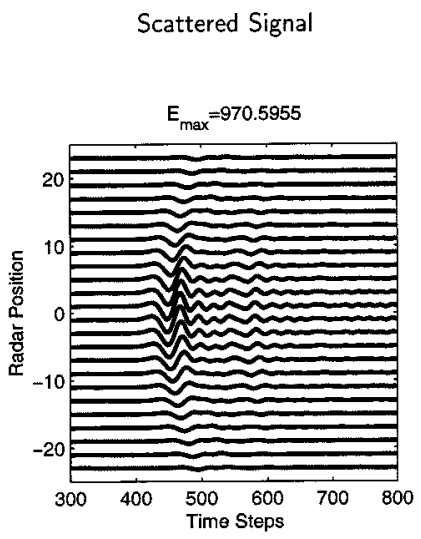

(b)
Fig. 5. Simulation results of a dielectric $\left(\epsilon_{r}=3\right)$ rectangular prism $\left(5 \times 5 \times 4 \mathrm{~cm}^{3}\right)$ buried $2.5 \mathrm{~cm}$ under the ground $\left(\epsilon_{r}=8\right)$. (a) The received and (b) the scattered signals recorded by the TR pair. The center frequency of the current signal on the transmitting dipole is $2000 \mathrm{MHz}$. The unit of radar position is $\Delta$, where $\Delta=2.5 \mathrm{~mm}$, and the unit of time steps is $\Delta t$, where $\Delta t=4.5 \mathrm{ps}$. The $\mathrm{T}$ and $\mathrm{R}$ are separated by $10.5 \mathrm{~cm}$, and both are $13 \mathrm{~cm}$ above the ground. The received signal in (a) is displayed in the time interval that the $\mathrm{S}$ signals arrive at the receiver, therefore windowing out the large $\mathrm{D}$ signal

to facilitate the detection of the desired $\mathrm{S}$ signal. Such isolation can be provided by placing perfectly conducting shield walls between and around the two antennas.

In addition to reducing the direct coupling to the receiver, there are two other reasons for shielding the antennas: First, shielding also reduces the coupling of the exterior noise to the receiver. Second, the transmitting and the receiving antennas become more directive with shield walls around them. For these reasons, it has been a popular technique to shield the antennas in practical [2] and computational GPR applications [8]. Examples of shielded GPR geometries are shown in Fig. 6(b) and (c).

Conducting shield walls alone are not completely beneficial since they cause large reflections and generate resonant fields. The resonance effects are observed as high-frequency oscillations on the total received signal. In practical applications, this problem is solved by mounting absorbers on the inner faces of the shield walls [23], as depicted in Fig. 6(d) and (e). Current research on absorbers [24],[25] constantly produces new absorbing materials with reduced thickness and high performance for practical use. The improved features of these novel materials provide high absorption in a small thickness, which, in turn, results in rapidly varying fields. Although the FDTD modeling of isolated absorbers is possible, it is almost impossible to accurately model the rapid fields inside the absorbers in a system-level simulation as discussed in this paper. Therefore, such high-performance physical absorbers are simulated using the PML ABC in this paper. That is, the PML ABC is implemented on the inner walls of the conducting shields inside the FDTD computational domain, in addition to the outer boundaries.

For the transmitter operating at a center frequency of $500 \mathrm{MHz}$ and a Yee cell size of $5 \mathrm{~mm}$, the thickness of the PML absorbers is selected as four cells. The PML absorbers are backed by the conducting walls of the shields. In addition, the sides of the PML absorbers (along their thicknesses) must also be covered by conducting shields, as illustrated in Fig. 6(d) and (e). Otherwise, elec-
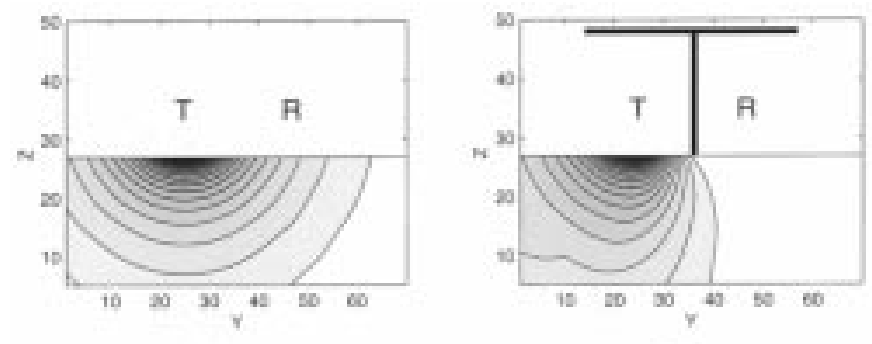

(a)

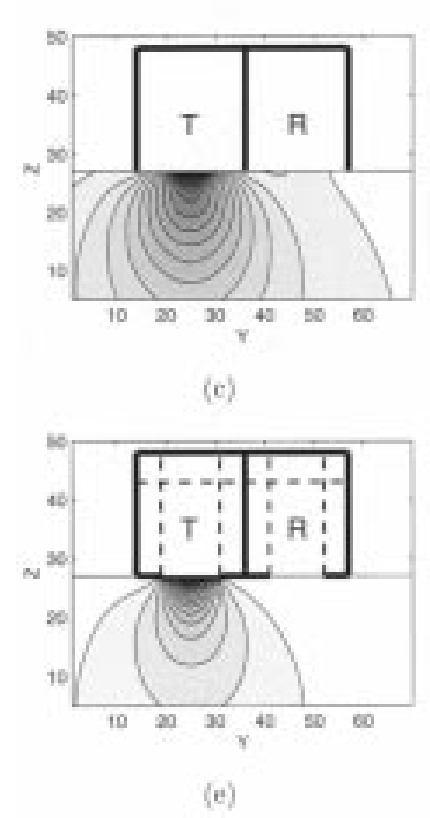

(b)

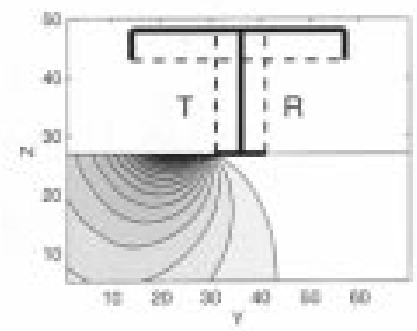

(d)

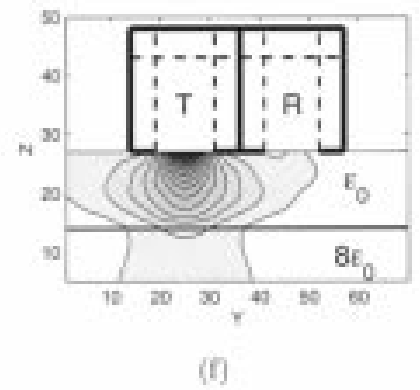

Fig. 6. Radiation patterns of five GPR models: (a) simple TR pair in free space (b) $\mathrm{SH} 1$ in free space, (c) $\mathrm{SH} 2$ in free space, (d) $\mathrm{SH} 3$ in free space, (e) $\mathrm{SH} 4$ in free space, and (f) $\mathrm{SH} 4$ over a homogeneous dielectric ground model with permittivity of $8 \epsilon_{0}$. The unit of both $y$ - and $z$-axes is $\Delta$, where $\Delta=5 \mathrm{~mm}$.

tromagnetic boundary conditions would be violated on these side surfaces of the PML absorbers. The conducting shields along the side walls of the PML absorbers produce large reflections. The conductivities of the PML absorbers are selected unusually large in order to minimize the total reflection, which is dominated by the reflection from these side walls. The PML absorbers inside the shields employ quadratic conductivity profile with a maximum value of $45.8 \mathrm{~S} / \mathrm{m}$, a value much larger than the typical conductivity values used in the PML ABCs placed on the borders of the FDTD computational domain.

The radiation patterns of five different GPR models are given in Fig. 6(a)-(e). These patterns are obtained by plotting

$$
\max _{n} E_{x}^{0, j, k, n}=\max _{n} E_{x}(i \Delta x=0, j \Delta y, k \Delta z, n \Delta t)
$$

which are the maxima of the $x$-component of the electric-field values at every point on a $y-z$ plane centered with respect to the radar unit. In Fig. 6(a)-(e), no ground model is implemented in the simulations and the $y-z$ plane, on which the pattern plots are given, is in free space. The radiation patterns given in Fig. 6(a)-(f) are normalized individually. The $y$ - and $z$-axes denote the $j$ and $k$ indices of the corresponding Yee cell in (10), where the size of a Yee cell is set as $5 \mathrm{~mm}$. For the simple TR pair, the large coupling to the receiver is observed in Fig. 6(a). 
The shield model presented in Fig. 6(b), which consists of two conducting sheets, separates the transmitter from the receiver and covers their top. The transmitting and the receiving dipoles are both placed at an elevation of $13 \mathrm{~cm}$ from the ground-air interface. The shield walls, which are $11 \mathrm{~cm}$ long in the $x$-direction, are placed $5 \mathrm{~cm}$ away from the dipole antennas, while the transmitter and the receiver are separated by $11 \mathrm{~cm}$ in the $y$-direction. In this work, the shield model shown in Fig. 6(b) is named SH1. Fig. 6(b) shows that SH1 provides a slight directivity toward the ground.

The second shield model, named SH2 and shown in Fig. 6(c), encloses the transmitter and the receiver in two chambers, leaving only the bottom faces open. The transmitter-receiver pair is again separated by $11 \mathrm{~cm}$ and the shield walls are again $11 \mathrm{~cm}$ long in the $x$-direction and $5 \mathrm{~cm}$ away the antennas. SH2 provides a better directivity toward the ground, due to its side walls' enclosing the transmitting antenna.

The inner faces of the models $\mathrm{SH} 3$ and SH4, illustrated in Fig. 6(d) and (e), respectively, are coated with four-cell-thick PML absorbers, i.e., SH3 is assembled by using PML ABC inside SH1, and SH4 is similarly constructed from SH2. Shield model $\mathrm{SH} 3$ produces a radiation pattern that is very similar to that of SH1, as depicted in Fig. 6(d). However, Fig. 6(e) demonstrates that $\mathrm{SH} 4$, in contrast to $\mathrm{SH} 2$, maintains a good directivity toward the ground. In addition to Fig. 6(a)-(e), Fig. 6(f) illustrates the radiation pattern of the GPR model in Fig. 6(e) over a homogeneous ground model with $8 \epsilon_{0}$ permittivity. Therefore, the $y-z$ plane in Fig. 6(f) extends into the ground. Fig. 6(f) demonstrates that the existence of the ground increases the coupling to the receiver, due to the reflections from the ground-air interface.

To demonstrate the effect of the PML absorbers inside the GPR models in Fig. 6, the reflection ratio of the incident signal is obtained at the transmitter location of SH4. Two simulations are performed, with the SH4 model present and absent, where the $x$-components of the electric-field variables at the transmitter locations are recorded for 1024 time steps. Then, the results of these simulations are subtracted from each other to obtain the signal reflected from the PML and conducting walls of SH4. The fast Fourier transform (FFT) of this difference signal is divided by the FFT of the signal obtained with no shield present, in order to calculate the electric-field reflection ratios with respect to frequency. Fig. 7 displays these reflection coefficients, obtained by model $\mathrm{SH} 4$, on a logarithmic scale. As demonstrated in Fig. 3(b), the energy of the source signal is predominantly carried by the frequency components less than $4 \mathrm{GHz}$. Fig. 7 presents the reflection coefficients for frequency components below $4 \mathrm{GHz}$ with a solid curve, while the values above $4 \mathrm{GHz}$ are plotted with a dashed curve. Fig. 7 illustrates that the electric-field reflection coefficients obtained with $\mathrm{SH} 4$ are less than $10^{-2}$ for the dominant frequency components. Therefore, the PML walls mounted on the inner walls of the shield model SH4 achieve a significant amount of absorption of the waves incident on them.

\section{SiMULATION RESUlTS}

To better illustrate the effects of the shield models, Fig. 8 displays the simulation results obtained with five different GPR

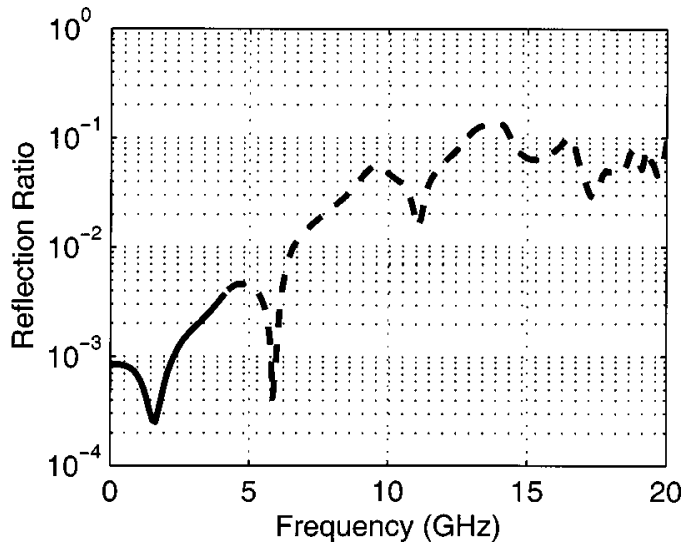

Fig. 7. The electric-field reflection ratios of shield model SH4 with respect to frequency.

models. In all of these simulations, $\Delta=5 \mathrm{~mm}, \Delta t=9 \mathrm{ps}$, and the GPR units operating at $f_{0}=500 \mathrm{MHz}$ are located $7.5 \mathrm{~cm}$ above the ground-air interface. A dielectric rectangular prism of $5 \times 5 \times 4 \mathrm{~cm}^{3}$ and $\epsilon_{r}=3$ is buried $15 \mathrm{~cm}$ under the ground and exactly under the GPR unit. The ground is modeled as a homogeneous dielectric half-space with a permittivity of $8 \epsilon_{0}$. The peak-to-peak amplitudes of the total and scattered signals are given in Table I, which also displays the ratios of these amplitude values for the five GPR models shown in Fig. 8(a)-(e).

Fig. 8 displays the problem geometries, scattered signals, and received signals for all GPR models introduced in Fig. 6. The scattered signals shown in Fig. 8 are obtained from the total received signals by subtracting the results of extra simulations performed in the absence of the scatterer, as described in Section III. It should be noted that these extra simulations are performed with exactly the same computational configuration as the simulations involving the buried target. The use of identical configurations in both simulations increases the accuracy of the scattered signals presented in Fig. 8. Since the waves emitted by the transmitter and reflected by the PML boundaries are present in both simulations, their subtraction yields the elimination of the largest PML reflections in the simulations. Moreover, the waves first reflected from the ground-air interface and then from the PML boundaries are also present in both simulations, and thus absent in the scattered signals. The largest noise present in the scattered-signal plots of Fig. 8(a)-(e) are due to the signals that are first scattered from the target and then reflected by the PML boundary. This noise signal is much smaller than the scattered signal that directly reaches the receiver, and thus, the scattered signal is dominated by the signals reflected from the target. The elimination of these PML reflections is important in certain GPR simulations, such as Fig. 8(a), where the waves reflected from the buried target are much smaller than the total received signal. If the PML reflection is comparable to or larger than the scattered waves, then it may not be possible to obtain the scattered signal from the simulation results.

Fig. 8(a) displays the results obtained when the simple TR pair is used, with no shields or absorbers employed. Although the scattered signal is slightly visible in the received signal just before the five-hundredth time step in Fig. 8(a), the total signal is 151 times larger than the the scattered signal, as displayed 
Scattered Signal
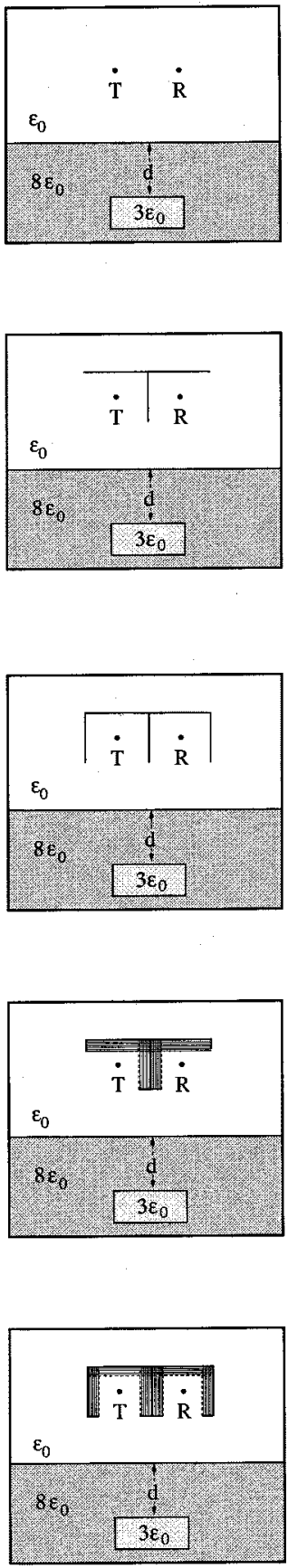

(a)

(b)

(c)

(d)
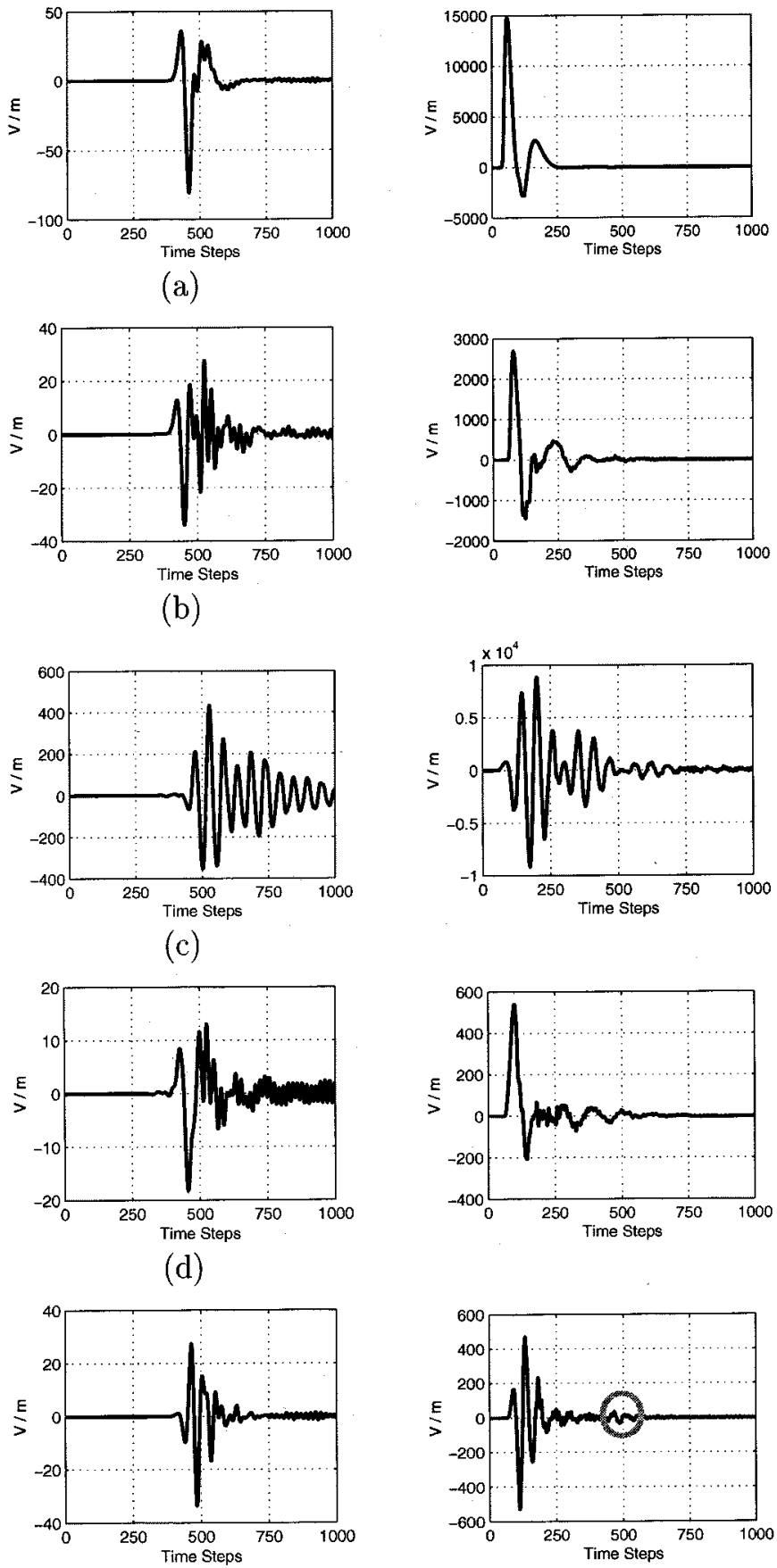

(e)

Fig. 8. Simulation results obtained with five different GPR models: (a) simple TR pair, (b) SH1, (c) SH2, (d) SH3, and (e) SH4. The center frequency of the transmitter is $500 \mathrm{MHz}$. A dielectric rectangular prism of $5 \times 5 \times 4 \mathrm{~cm}^{3}$ is buried $15 \mathrm{~cm}$ under the ground. The GPR unit is located exactly above the dielectric prism. The unit of time steps is $\Delta t$, where $\Delta t=9 \mathrm{ps}$.

in the bottom row of Table I. Considering the additional noise factor in the practical GPR applications, it is very difficult to detect such a weak scattered signal in the large total signal.

Table I displays that the shield model SH1, depicted in Fig. 8(b), reduces the coupling to the receiver from 17602 $\mathrm{V} / \mathrm{m}$ to $4139 \mathrm{~V} / \mathrm{m}$. However, SH1 also scales the scattered-field amplitude down by almost 50\%. Moreover, the signal received by SH1 has a relatively larger tail than the received signal of the simple TR pair. These two factors constitute a disadvantage for the detection of shallow buried targets. The scattered signal is hardly visible in the received signal shown in Fig. 8(b)

Fig. 8(c) demonstrates that the resonant fields due to the perfectly conducting shield walls are dominant when $\mathrm{SH} 2$ is employed. Table I suggests that the amplitude ratio of the received and the scattered signals is reduced to 23 by using $\mathrm{SH} 2$. However, the received signal in Fig. 8(c) contains large oscillations 
TABLE I

PEAK-TO-PEAK AMPlitudes of THE SCATTERED AND THE TOTAL RECEIVED SIGNALS AND THEIR RATIOS FOR FIVE GPR MODELS

\begin{tabular}{c||l|l|l|l|l}
\hline \multicolumn{1}{c||}{} & \multicolumn{5}{c}{ GPR Model } \\
\hline & TR Pair & SH1 & SH2 & SH3 & SH4 \\
\hline \hline$|S|(\mathrm{V} / \mathrm{m})$ & 116 & 61 & 785 & 31 & 61 \\
\hline$|D+G+S|(\mathrm{V} / \mathrm{m})$ & 17,602 & 4,139 & 18,020 & 747 & 999 \\
\hline$\underline{|D+G+S|}$ & 151.3 & 67.2 & 23.0 & 23.9 & 16.4 \\
\hline$|S|$ & & & & & \\
\hline
\end{tabular}

in its late periods, which renders the detection of the scattered signal very difficult.

Fig. 8(d) presents the results obtained using SH3. In Fig. 8(d), the $\mathrm{S}$ signal is not visible on the total signal, due to the large tail of the total signal. However, when shield model SH4 is used, the scattered signal is observed on the received signal, as seen in Fig. 8(e). The interval where the scattered signal can be observed is marked (with a circle) on the total signal. Fig. 8(e) also illustrates the relatively smaller tail of the total received signal, easing the detection of the target.

In Table I, the amplitude ratio of the total and scattered signals is given as 23.9 for $\mathrm{SH} 3$, while the same ratio is 67.2 for SH1. Since the only difference between models SH1 and SH3 is the PML coating on the inner faces of the shield walls, it is possible to conclude that these absorbers decrease the amplitude ratio and facilitate the detection of the scattered signal. Table I also displays the peak-to-peak amplitude ratio as 16.4 for shield model SH4. SH4 is the most satisfactory shield model presented in this paper, not only with the lowest amplitude ratio but also with the small tail of the total signal.

The advantages of SH4 are further elaborated with the B-scan results presented in Fig. 9(a) and (b), where the dielectric prism is buried 10 and $15 \mathrm{~cm}$ deep, respectively. Except for the depth of the target, all other parameters of the simulations are exactly the same as those of Fig. 8(e). Careful examination of Fig. 9 reveals that shield model SH4, supported by PML absorbers, successfully weakens the direct signal coupling from the transmitter to the receiver. When the total signals are zoomed after the three-hundredth time step in order to window out the direct signals, the scattered signals become visually detectable in Fig. 9.

Conducting targets are in general stronger scatterers than dielectric targets. To demonstrate that SH4 performs even better for conducting targets, the dielectric prism of Fig. 9 is replaced by a perfectly conducting rectangular prism, keeping all the other parameters unchanged. Fig. 10 displays the B-scan results when the conducting prism is buried 10 and $15 \mathrm{~cm}$ under the ground-air interface. The scattered signals are clearly observed in the total received signals, without any windowing in time, establishing that the energies of the scattered signals are comparable with the energies of the direct signals coupled to the receiver.

\section{CONCLUSION}

In this paper, 3-D GPR simulations are carried out using the FDTD method. Ground is modeled as a homogeneous medium,

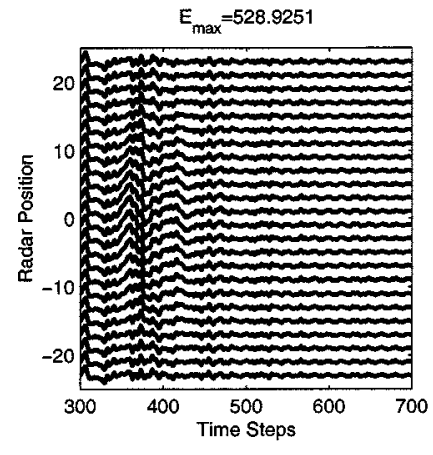

(a)

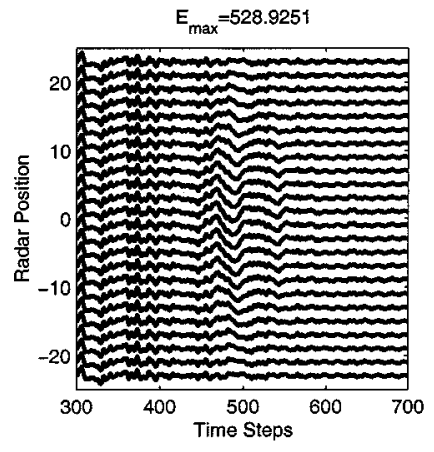

(b)
Fig. 9. B-scan signals recorded by the GPR model SH4 moving above a dielectric rectangular prism buried (a) 10 and (b) $15 \mathrm{~cm}$ underground. The unit of radar position is $\Delta$, where $\Delta=5 \mathrm{~mm}$, and the unit of time steps is $\Delta t$, where $\Delta t=9 \mathrm{ps}$.

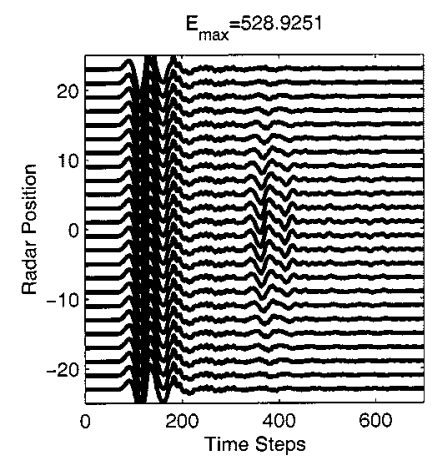

(a)

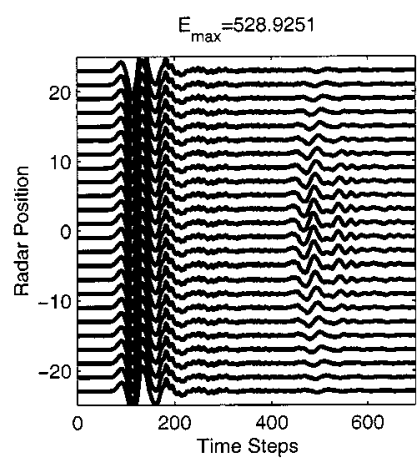

(b)
Fig. 10. B-scan signals recorded by the GPR model SH4 moving above a conducting rectangular prism buried (a) 10 and (b) $15 \mathrm{~cm}$ underground. The unit of radar position is $\Delta$, where $\Delta=5 \mathrm{~mm}$, and the unit of time steps is $\Delta t$, where $\Delta t=9 \mathrm{ps}$.

and the targets are modeled as dielectric and conducting rectangular prisms. The computational space is terminated by layered PML ABC, which matches the air, the ground regions, and the interface between them. A realistic GPR unit is modeled by a pair of single-cell receiving and transmitting antennas, isolated by perfectly conducting shield walls coated with absorbers. The absorbers are not selected as lossy physical materials. Instead, four-cell-thick PML absorbers are placed inside the shield walls to absorb the waves excited by the transmitter and reflected from the ground, target, or exterior shield walls.

The GPR configuration with one transmitting and one receiving antenna experiences a large coupling from the transmitter to the receiver. If this coupling is not prevented, then the receiver is blinded by the direct coupling, since this signal firmly dominates the total received signal. In this case, although the total signal is composed of three signals- the coupling from the transmitter, the signal reflected from the ground, and the signal scattered from the target-it is not possible to detect the desired signal, which is the smallest of the three signals, the scattering from the buried target. To overcome this difficulty, the transmitting and the receiving antennas are isolated by various shield models, constructed by conducting walls. However, the majority of these shield models induce resonant fields, observed as large and slowly decaying oscillations, due to the large reflections 
from the inner walls of the shields. In practice, thin microwave absorbers, composed of many thinner slabs of different materials, are employed to absorb the waves incident on the shield walls and prevent the resonance effects, but such material absorbers are computationally expensive to model in the FDTD grid. Instead, the function of the physical absorbers is simulated by using PML absorbers inside the computational domain, on the inner faces of the shield walls. It is observed that the application of PML absorbers elevates the performances of all of the shield models, compared to their performances without absorbers. The GPR model that has the best performance is used to simulate various scenarios with targets of arbitrary conductivity, permittivity, and depth. Simulation results demonstrate that the scattered signal is no longer a very small portion of the total received signal, and that it is even possible to visually detect the buried target in many scenarios.

\section{REFERENCES}

[1] K. S. Yee, "Numerical solution of initial boundary value problems involving Maxwell's equations in isotropic media," IEEE Trans. Antennas Propagat., vol. 14, pp. 302-307, May 1966.

[2] D.J. Daniels, Surface-Penetrating Radar. London, U.K.: Institute of Electrical Engineers, 1996.

[3] Proc. Detection and Remediation Technologies for Mines and Minelike Targets III, A. C. Dubey, J. F. Harvey, and J. T. Broach, Eds., Orlando, FL, Apr. 1998.

[4] Proc. 7th Int. Conf. Ground-Penetrating Radar (GPR'98), Lawrence, KS, May 1998.

[5] M. Moghaddam, W. C. Chew, B. Anderson, E. J. Yannakakis, and Q. H. Liu, "Computation of transient electromagnetic waves in inhomogeneous media," Radio Sci., vol. 26, no. 1, pp. 265-273, Jan.-Feb. 1991.

[6] M. Moghaddam, E. J. Yannakakis, W. C. Chew, and C. Randall, "Modeling of the subsurface interface radar," J. Electromagn. Waves Appl., vol. 5, no. 1, pp. 17-39, 1991.

[7] J. M. Bourgeois and G. S. Smith, "A fully three-dimensional simulation of a ground-penetrating radar: FDTD theory compared with experiment," IEEE Trans. Geosci. Remote Sensing, vol. 34, pp. 36-44, Jan. 1996.

[8] _ - "A complete electromagnetic simulation of the separated-aperture sensor for detecting buried land mines," IEEE Trans. Antennas Propagat., vol. 46, pp. 1419-1426, Oct. 1998.

[9] P. Luneau and G. Y. Delisle, "Underground target probing using FDTD," in Proc. 1996 IEEE AP-S Int. Symp. URSI Radio Science Meeting, Baltimore, MD, July 1996, pp. 1664-1667.

[10] U. Oğuz and L. Gürel, "Subsurface-scattering calculations via the 3D FDTD method employing PML ABC for layered media," in 1997 IEEE AP-S Int. Symp. URSI Radio Science Meeting, Montréal, PQ, Canada, July 1997.

[11] F. L. Teixeria, W. C. Chew, M. Straka, and M. L. Oristaglio, "Finite-difference time-domain simulation of ground-penetrating radar on dispersive, inhomogeneous, and conductive soils," IEEE Trans. Geosci. Remote Sensing, vol. 36, pp. 1928-1937, Nov. 1998.

[12] J. P. Berenger, "A perfectly matched layer for the absorption of electromagnetic waves," J. Comput. Phys., pp. 185-200, Oct. 1994.

[13] W. C. Chew and W. H. Weedon, "A 3D perfectly matched medium from modified Maxwell's equations with stretched coordinates," Microwave Opt. Tech. Lett., vol. 7, no. 13, pp. 599-604, Sept. 1994.

[14] D. S. Katz, E. T. Thiele, and A. Taflove, "Validation and extension to three dimensions of the Berenger PML absorbing boundary condition for FD-TD meshes," IEEE Microwave Guided Wave Lett., vol. 4, pp. 268-271, Aug. 1995.

[15] J. B. Verdu, R. Gillard, K. Moustadir, and J. Citerne, "An extension of the PML technique to the FDTD analysis of multilayer planar circuits and antennas," Microwave Opt. Tech. Lett., vol. 10, no. 6, pp. 323-327, Dec. 1995.

[16] S. D. Gedney, "An anisotropic PML absorbing media for FDTD simulation of fields in lossy dispersive media," Electromagnetics, pp. 399-415, July/Aug. 1996.

[17] D. Johnson, C. Furse, and A. Tripp, "Application and optimization of the perfectly matched layer boundary condition for geophysical simulations," in Microwave Opt. Tech. Lett., Oct. 2000, to be published.
[18] L. Gürel and U. Oğuz, "Simulations of ground-penetrating radars over lossy and heterogeneous grounds," IEEE Trans. Geosci. Remote Sensing, vol. 39, June 2001.

[19] — , "Three-dimensional FDTD modeling of a ground-penetrating radar," IEEE Trans. Geosci. Remote Sensing, vol. 38, pp. 1513-1521, July 2000 .

[20] — "Employing PML absorbers in the design and simulation of ground penetrating radars," in 1999 IEEE AP-S Int. Symp. USNC/URSI National Radio Science Meeting, Orlando, FL, July 1999, pp. 1890-1893.

[21] A. Taflove, Computational Electrodynamics: The Finite-Difference Time-Domain Method. Norwood, MA: Artech House, 1995.

[22] D. N. Buechler, D. H. Roper, C. H. Durney, and D. A. Christensen, "Modeling sources in the FDTD formulation and their use in quantifying source and boundary condition errors," IEEE Trans. Microwave Theory Tech., vol. 43, pp. 810-814, Apr. 1995.

[23] R. V. d. Jongh, L. P. Ligthart, I. V. Kaploun, and A. D. Schukin, "Design and analysis of new GPR antenna concepts," in Proc. 7th Int. Conf. Ground-Penetrating Radar (GPR'98), Lawrence, KS, May 1998.

[24] Y. Jiang and A. Q. Martin, "The design of microwave absorbers with high-order hybrid finite element method," in Proc. 1999 IEEE AP-S Int. Symp. USNC/URSI National Radio Science Meeting, Orlando, FL, July 1999, pp. 2622-2625.

[25] H. Mosallaei and Y. Rahmat-Samii, "RCS reduction in planar, cylindrical, and spherical structures by composite coatings using genetic algorithms," in Proc. 1999 IEEE AP-S Int. Symp. USNC/URSI National Radio Science Meeting, Orlando, FL, July 1999, pp. 438-441.

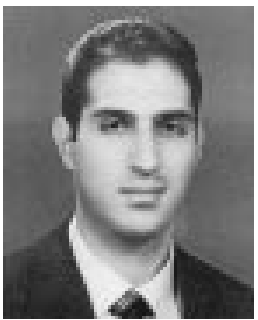

Uğur Oğuz was born in Ankara, Turkey, in 1973. He received the B.Sc. and M.Sc. degrees in electrical engineering from Bilkent University, Ankara, Turkey, in 1994 and 1997, respectively.

From August 1997 to October 1998, he served in the Turkish Army, working as a Database Manager Since November 1998, he has been a Research Engineer in the Department of Electrical and Electronics Engineering, Bilkent University. His research interests include time-domain methods in computational electromagnetics and their applications to geophys-

ical problems.

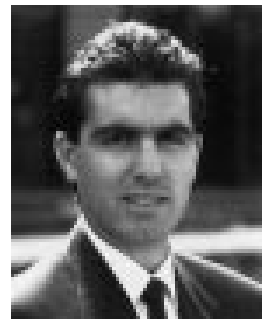

Levent Gürel (S'87-M'92-SM'97) was born in İzmir, Turkey, in 1964. He received the B.Sc. degree from the Middle East Technical University, Ankara, Turkey, in 1986 and the M.S. and Ph.D. degrees from the University of Illinois at Urbana-Champaign (UIUC) in 1988 and 1991, respectively, all in electrical engineering.

He joined the T. J. Watson Research Center, IBM Corporation, Yorktown Heights, NY, in 1991, where he worked as a Research Staff Member on the electromagnetic compatibility problems related to electronic packaging, the use of microwave processes in the manufacturing and testing of electronic circuits, and the development of fast solvers for interconnect modeling. He became an Associate Professor in 1993. Since 1994, he has been a Faculty Member in the Department of Electrical and Electronics Engineering, Bilkent University, Ankara. He was a Visiting Associate Professor at the Center for Computational Electromagnetics of the UIUC for one semester in 1997. His research interests include the development of fast algorithms for computational electromagnetics and the application thereof to scattering and radiation problems involving large and complicated scatterers, antennas and radars, frequency-selective surfaces, and high-speed electronic circuits. He is also interested in the theoretical and computational aspects of electromagnetic compatibility and interference analyses. Ground-penetrating radars and other subsurface-scattering applications are also among his current research interests.

Dr. Gürel is currently Chairman of the AP/MTT/ED/EMC Chapter of the IEEE Turkey Section. He has published several papers in IEEE journals, actively attended numerous IEEE symposia, and served as a reviewer for various IEEE journals. 